EPJ Web of Conferences 66, 02052 (2014)

DOI: 10.1051/epjconf/ 20146602052

(C) Owned by the authors, published by EDP Sciences, 2014

\title{
Tensor correlations probed by electroweak responses
}

\author{
W. Horiuchi ${ }^{1, a}$ and Y. Suzuki $i^{2,3}$ \\ ${ }^{1}$ Department of Physics, Hokkaido University, Sapporo 060-0810, Japan \\ ${ }^{2}$ Department of Physics, Niigata University, Niigata 950-2181, Japan \\ ${ }^{3}$ RIKEN Nishina Center, RIKEN, Wako 351-0198, Japan
}

\begin{abstract}
In this contribution, we present our recent study on the electroweak response functions with realistic nuclear forces and discuss their relation to tensor correlations. The observed resonance energies and widths of the negative-parity levels are all in fair agreement with those calculated from both the spin-dipole and electric-dipole strength functions. Spin-dipole sum rules are discussed in relation to tensor correlations in the ground state of ${ }^{4} \mathrm{He}$. Possible observables are suggested to probe the tensor correlations.
\end{abstract}

\section{Introduction}

Nuclear response functions for electroweak interactions provide us with important information on the resonant and continuum structure of the nuclear system as well as the detailed property of the underlying interactions. Recently the present authors and Arai have presented an ab initio calculation for the photoabsorption of ${ }^{4} \mathrm{He}$ [1]. In the energy region around $26 \mathrm{MeV}$, photoabsorption reaction occurs mainly through the electric dipole transition. That paper shows that the experimental data above $30 \mathrm{MeV}$ is reproduced very well, and the one-pion-exchange terms in the nucleon-nucleon potential, especially the tensor term, are essential in accounting for the energy-weighted sum rule. Here we extend the discussion to the spin-dipole (SD) response function [2]. The operator belongs to the first-forbidden transition induced by the weak interaction and is expected to play a significant role for neutrino- ${ }^{4} \mathrm{He}$ reactions.

Theoretically it is well known that the ground state of ${ }^{4} \mathrm{He}$ contains the $D$-state (or the total spin $S=2$ state) probability by about $14 \%$, which is of course due to the tensor force. We will discuss the effect of the tensor correlation on the SD excitations and possibility of observing the correlation. It should be noted that the SD excitation is here described based on the accurate ground-state wave function of ${ }^{4} \mathrm{He}[3,4]$. We also note that this study will serve fundamental data on the neutrino- ${ }^{4} \mathrm{He}$ reaction cross section in stars by integrating the SD strength functions weighted by the neutrino energy distribution produced by the core collapse star.

In the next section, we briefly explain our theoretical prescriptions for calculating the strength functions of the four nucleon system. In Section 3 we present the calculated SD strength functions and discuss the effect of the tensor correlation on them. Conclusions are given in Section 4.

\footnotetext{
ae-mail: whoriuchi@nucl.sci.hokudai.ac.jp
} 


\section{Spin-dipole response functions}

The Hamiltonian of four-nucleon system consists of two- and three-nucleon forces. As a nucleonnucleon potential, we employ the Argonne $v 8^{\prime}$ [5] (AV8') and G3RS [6] potentials which contain central, tensor and spin-orbit components. A phenomenological three-body interaction (3NF) [7] is used that reproduces the binding energies of the three- and four-nucleon bound states. The wave function is expressed in terms of a linear combination of the basis functions whose orbital part is given by the correlated Gaussian with two global vectors [8]. The variational parameters are selected by using the stochastic variational method $[9,10]$.

A SD response function is given by

$$
S(p, \lambda, E)=\mathcal{S}_{f \mu}\left|\left\langle\Psi_{f}\left|\sum_{i=1}^{N}\left[\boldsymbol{\rho}_{i} \times \sigma_{i}\right]_{\lambda \mu} T_{i}^{p}\right| \Psi_{0}\right\rangle\right|^{2} \delta\left(E_{f}-E_{0}-E\right), \quad \text { with } \quad \boldsymbol{\rho}_{i}=\boldsymbol{r}_{i}-\boldsymbol{x}_{N},
$$

where $\boldsymbol{r}_{i}$ is $i$ th nucleon coordinate, $\boldsymbol{x}_{N}$ is the center-of-mass coordinate of the $N$-nucleon system, and $\sigma_{i}$ is $i$ th nucleon spin. The square bracket $\left[\rho_{i} \times \sigma_{i}\right]_{\lambda \mu}$ denotes the angular momentum coupling of two vectors to that operator specified by $\lambda \mu$. The superscript $p$ of $T_{i}^{p}$ distinguishes different types of isospin operators, isoscalar (IS) and isovector (IV0). The $\Psi_{0}\left(\Psi_{f}\right)$ is the ground (final) state of ${ }^{4} \mathrm{He}$ with the energy $E_{0}\left(E_{f}\right)$, and $\mathcal{S}_{f \mu}$ denotes a summation over all final states and $\mu$. For the continuum wave function, we use a square-integrable $\left(\mathcal{L}^{2}\right)$ basis function which does not satisfy a proper boundary condition of continuum state. To treat it properly, we use the complex scaling method (CSM) which is a widely used method for calculating the strength function in a nuclear system [13]. The accuracy of the CSM calculation crucially depends on how completely the $\mathcal{L}^{2}$ basis functions are prepared. We attempt at constructing the basis paying attention to two points: the sum rule of the electroweak strength functions and the decay channels $[1,2]$.

\section{Results and discussions}

\subsection{Spin-dipole strength functions and spectrum}
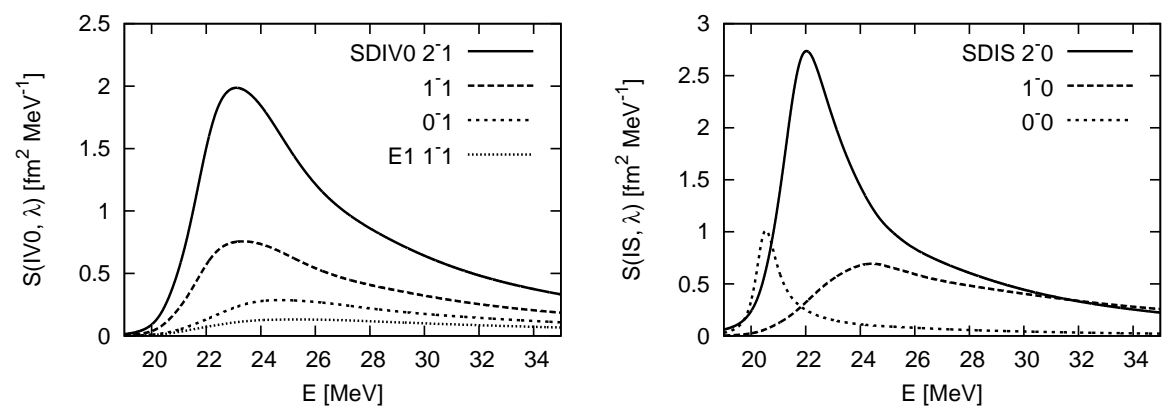

Figure 1. Left: Isovector SD strength functions of IV0 type and $E 1$ strength function for ${ }^{4} \mathrm{He}$ as a function of excitation energy. The AV8' $+3 \mathrm{NF}$ potential is used. Right: The same plot as the left but isoscalar SD strength functions of IS type. The figures are plotted based on Ref. [2].

The left panel of Figure 1 plots the SD strength functions of IV0 type with the AV8' $+3 \mathrm{NF}$ potential. For the sake of comparison, the electric dipole $(E 1)$ strength function is also plotted by 
choosing the $E 1$ operator as $\sum_{i=1}^{N} \rho_{i \mu} \frac{1}{2}\left(1-\tau_{z}(i)\right)$. As seen in the figure, the three SD strength functions show narrower widths at their peaks than the $E 1$ strength function. Moreover their peak positions including the $E 1$ case well correspond to the observed excitation energies of the four $T=1$ negativeparity states of ${ }^{4} \mathrm{He}$ [11]. The results with the G3RS potential give similar results.

We display in the right panel of Fig. 1 the IS SD strength functions that reflect the $J^{\pi} T=\lambda^{-} 0$ continuum states of ${ }^{4} \mathrm{He}$. These IS SD strength functions, especially for the $0^{-}$and $2^{-}$cases, show much narrower distribution than the IV0 strength functions. These peak energies again appear to correspond to the observed $T=0$ negative-parity levels in ${ }^{4} \mathrm{He}$. A close comparison between the IS and IV0 strength functions indicates that the $0^{-}$case is noteworthy compared to the $1^{-}$and $2^{-}$cases in that the difference in the peak positions of the same $J^{-}$becomes much larger. As discussed in Refs. [3, 4], the reason for this is understood by analyzing the role played by the tensor force.

All the SD and $E 1$ strength functions exhibit some common feature: They all have one peak, though the width of the strength distribution depends on the multipolarity $\lambda$ and the isospin $T$. It looks quite reasonable to identify the peak as a resonance. The resonance energy may be identified with the energy where the peak is located. We also estimate the decay width of the resonance by the difference of two excitation energies at which the strength becomes half of the maximum strength at the peak. The agreement between theory and experiment is very satisfactory. The average deviation of the calculated resonance energies from experiment is less than $0.4 \mathrm{MeV}$ for ${ }^{4} \mathrm{He}$ despite the fact that most of their widths are larger than $5 \mathrm{MeV}$. The estimated width is also in reasonable agreement with experiment.

\subsection{Spin-dipole sum rule}

Here we discuss the non energy-weighted sum rule (NEWSR) for the SD operator. The use of the closure relation enables us to express the NEWSR to the expectation value of the operator $\sum_{\mu} O_{\lambda \mu}^{p \dagger} O_{\lambda \mu}^{p}$ with respect to the ground state $\Psi_{0}$. It is convenient to express that operator as a scalar product of the space-space and spin-spin tensors, $Q_{(\kappa) 0}^{p}=\sum_{i, j=1}^{A}\left(\left[\rho_{i} \times \rho_{j}\right]_{\kappa} \cdot\left[\sigma_{i} \times \sigma_{j}\right]_{\kappa}\right) T_{i}^{p^{\dagger}} T_{j}^{p}$, where the rank $\kappa$ can be 0,1 , and 2 . With use of the above equation, the expression of the NEWSR is

$$
m_{0}(p, \lambda)=\int_{0}^{\infty} S(p, \lambda, E) d E=\sum_{\kappa=0}^{2} U_{\lambda_{k}}\left\langle Q_{(\kappa) 0}^{p}\right\rangle
$$

In order to check that the NEWSR is satisfied, we compare $m_{0}(p, \lambda)$ that is calculated separately with the middle and left hand side of Eq. (2). The two different ways of calculating the sum rules give virtually the same result for both cases of $\mathrm{AV}^{\prime}+3 \mathrm{NF}$ and G3RS+3NF interactions. The deviation is less than $1 \%$. The perfect agreement confirms that the basis functions prepared for the description of the SD excitation are sufficient enough to account for all the strength in the continuum. Among the three expectation values of $\left\langle Q_{(\kappa) 0}^{p}\right\rangle$ in Eq. (2), the $\kappa=0$ term gives a dominant contribution to the NEWSR because the major component of the ground state of ${ }^{4} \mathrm{He}$ is $S=0$ and it has a non-vanishing expectation value only for $Q_{(0) 0}^{p}$. In this limiting case $m_{0}(p, \lambda)$ is proportional to $U_{\lambda 0}$. Therefore the $\lambda$ dependence of the NEWSR turns out to be $1: 3: 5$ for $\lambda=0,1,2$, independently of $p$. The deviation from this ratio is due to the contributions of other $Q_{(\kappa) 0}^{p}$ terms, especially the $\kappa=2$ term. Since the admixture of the $S=2$ component is primarily determined by the tensor force, the deviation reflects the tensor correlations in the ground state. The term with $\kappa=2$ is particularly interesting because it contains the tensor operator characteristic of the one-pion-exchange potential. Since $\left\langle Q_{(2) 0}^{p}\right\rangle$ is negative for $p=$ IS, the ratio further increases from $1: 3: 5$, which is approximately $1: 4: 7$, whereas it is positive for $p=\operatorname{IV} 0$ and the ratio approximately reduces to $1: 2: 4$. 
As discussed above, $\left\langle Q_{(\kappa) 0}^{p}\right\rangle$ plays a central role to determine the NEWSR for the SD strength functions. Inverting the right hand side of Eq. (2) makes it possible to express $\left\langle Q_{(\kappa) 0}^{p}\right\rangle$ as a sum, over the multipole $\lambda$, of the NEWSR, $\left\langle Q_{(\kappa) 0}^{p}\right\rangle=\sum_{\lambda=0}^{2} U_{k \lambda}^{-1} m_{0}(p, \lambda)$, where $U^{-1}$ is the inverse matrix of $U$. If the NEWSR for all $\lambda$ are experimentally measured, the above equation indicates that $\left\langle Q_{(\kappa) 0}^{p}\right\rangle$ for all $\kappa$ can be determined from experiment.

\section{Summary}

We have investigated the electroweak responses of ${ }^{4} \mathrm{He}$ based on the four-body calculation using a realistic nuclear force. The wave function of the ground state is obtained accurately using an explicitly correlated basis. The spin-dipole (SD) excitation is built on the ground state of ${ }^{4} \mathrm{He}$ that is described accurately with use of explicitly correlated Gaussian bases. The continuum states including two- and three-body decay channels are described with aid of the complex scaling method.

Experimental data that can directly be compared to the calculation are presently only the resonance parameters of the negative-parity levels of $A=4$ nuclei. Both the resonance energies and widths deduced from the SD and electric-dipole strength functions or the eigenvalues of the complex-scaled Hamiltonian are all in fair agreement with experiment. This success is never trivial considering that most of the resonances among 15 levels have broad widths larger than $5 \mathrm{MeV}$.

The non energy-weighted sum rule (NEWSR) of the SD strength function is investigated by relating it to the expectation values of three scalar products of the space-space and spin-spin tensors with respect to the ground state of ${ }^{4} \mathrm{He}$. It turns out that our model space satisfies the NEWSR for each SD operator perfectly. The tensor operator of rank $2, Q_{(2) 0}^{p}$, is sensitive to the $D$-state correlation in the ground state induced by the tensor force, and it is mainly responsible for distorting the ratio of the NEWSRs for the multipolarity $\lambda=0,1,2$ from the uncorrelated ratio of $1: 3: 5$. An experimental observation of this ratio is desirable since it may lead us to reveal the degree of tensor correlations in the ground state.

The work is supported in part by Grants-in-Aid for Scientific Research (Nos. 21540261, 25800121) of Japan Society for Promotion of Science.

\section{References}

[1] W. Horiuchi, Y. Suzuki, and K. Arai, Phys. Rev. C 85, 044606 (2012).

[2] W. Horiuchi and Y. Suzuki, Phys. Rev. C 87, 034001 (2013).

[3] W. Horiuchi and Y. Suzuki, Phys. Rev. C 78, 034305 (2008).

[4] W. Horiuchi and Y. Suzuki, Few-Body Syst., in press, DOI 10.1007/s00601-012-0495-y.

[5] B. S. Pudliner et al., Phys. Rev. C 56, 1720 (1997).

[6] R. Tamagaki, Prog. Theor. Phys. 39, 91 (1968).

[7] E. Hiyama, B. F. Gibson, and M. Kamimura, Phys. Rev. C 70, 031001(R) (2004).

[8] Y. Suzuki, W. Horiuchi, M. Orabi, and K. Arai, Few-Body Syst. 42, 33 (2008).

[9] K. Varga and Y. Suzuki, Phys. Rev. C 52, 2885 (1995).

[10] Y. Suzuki and K. Varga, Sthochastic Variational Approach to Quantum-Mechanical Few-Body

Problems, Lecture Notes in Physics, (Springer, Berlin, 1998), Vol. m54.

[11] D. R. Tilley, H. R. Weller, and G. M. Hale, Nucl. Phys. A 541, 1 (1992).

[12] J. Mitroy et al., Rev. Mod. Phys. 85, 693 (2013).

[13] S. Aoyama, T. Myo, K. Katō, and K. Ikeda, Prog. Theor. Phys. 116, 1 (2006). 